\title{
GLAD!
}

Revue sur le langage, le genre, les sexualités

11 | 2021

Archives, genre, sexualités, discours

\section{Toutes pour une, une pour toustes ? Ou : que faire du masculin à valeur générique?}

Les genres récrits : chronique $n^{\circ} 9$

All for One, One for All? Or: What to Do with Generically Intended Masculines?

\section{Daniel Elmiger}

\section{OpenEdition}

\section{Journals}

Édition électronique

URL : https://journals.openedition.org/glad/3619

DOI : $10.4000 /$ glad.3619

ISSN : 2551-0819

Éditeur

Association GSL

Référence électronique

Daniel Elmiger, «Toutes pour une, une pour toustes ? Ou : que faire du masculin à valeur générique ?», GLAD! [En ligne], 11 | 2021, mis en ligne le 20 décembre 2021, consulté le 29 janvier 2022. URL : http:// journals.openedition.org/glad/3619; DOI : https://doi.org/10.4000/glad.3619

Ce document a été généré automatiquement le 29 janvier 2022.

\section{c) () $\Theta$}

La revue GLAD! est mise à disposition selon les termes de la Licence Creative Commons Attribution Pas d'Utilisation Commerciale - Pas de Modification 4.0 International. 


\section{Toutes pour une, une pour toustes? $\mathrm{Ou}$ : que faire du masculin à valeur générique?}

Les genres récrits : chronique $n^{\circ} 9$

All for One, One for All? Or: What to Do with Generically Intended Masculines?

Daniel Elmiger

\section{Identification du noyau du problème ${ }^{1}$}

1 Dans la dernière chronique, j'ai abordé la pluralité des désignations pour ce que je résume, un peu maladroitement, sous «langue non sexiste / inclusive ». Pour ne reprendre que les adjectifs et autres qualificatifs, on peut trouver (p. ex. à propos de la langue ou la communication): épicène, égalitaire, féministe, neutre, non binaire, non discriminatoire, non sexiste, sans stéréotype, transgenre - et évidemment inclusif/inclusive, désignation devenue majoritaire à partir du milieu des années $2010^{2}$. Entretemps, les questions lexicales, prédominantes encore dans les années 1990 (on a vraiment pu se disputer pour savoir s'il était légitime de dire la juge, la capitaine ou la ministre), sont largement réglées. Il subsiste un certain nombre de variantes ${ }^{3}$, mais la question centrale est désormais la suivante ${ }^{4}$ :

- Que faire des formes masculines à valeur générique?

La recherche montre qu'elles suscitent largement des représentations biaisées ${ }^{5}$ et qu'elles peuvent également avoir des répercussions sur les pratiques sociales, p. ex. lorsqu'une offre d'emploi est formulée au seul masculin. Ces résultats (ou les conséquences qui en sont tirées) ne sont pas partagés par celles et ceux qui sont de l'avis que le masculin à valeur générique a tout à fait sa place dans la grammaire et les usages et que sa remise en question crée plus de problèmes qu'elle n'en résout. 


\section{La généricité comme règle, comme jeu inférentiel ou comme négociation?}

3 La généricité du genre masculin (par rapport à des formes féminines) pose des problèmes théoriques et pratiques intéressants ${ }^{6}$. Est-ce un fait de sémantique lexicale ou le résultat d'une négociation en interaction? Et quel rôle joue, du point de vue pragmatico-sémantique, le calcul inférentiel permettant de distinguer entre différents types d'usage?

En simplifiant un peu, on peut esquisser trois cas de figure :

- Soit les deux fonctions «spécifique » et "générique » sont inscrites dans la grammaire du français et l'utilisation générique du masculin fait en quelque sorte partie de chaque nom commun de personne masculin qui a une forme féminine correspondante. Certes, en contexte, il s'agit parfois de déterminer si le(s) lecteur(s) désigne un ou des homme(s) spécifique(s) ou, de façon générique, une personne indéterminée ou un groupe mixte ${ }^{7}$, mais les deux fonctions du masculin sont inscrites dans le système de la langue.

- Si l'on écarte l'idée selon laquelle la fonction générique relève d'une règle de grammaire fixe, le jeu des inférences (en linguistique pragmatique, on parlerait aussi d'implicatures conversationnelles) peut alors concerner tout nom commun de personne, qu'il soit masculin ou féminin, car dans les deux cas, il peut y avoir usage spécifique ou générique.

- Enfin, au jeu des règles grammaticales et aux inférences pragmatiques peuvent s'ajouter des conventions ou négociations ad hoc, déterminées dans l'interaction. Elles peuvent alors être fixées par le sujet encodeur (parlant ou écrivant), p. ex. au moyen d'une note de bas de page expliquant comment les formes masculines - ou féminines - doivent être entendues - ou alors par son décodeur qui récuserait une telle convention (p. ex. en disant « je ne me sens pas incluse dans cette formulation »).

Récemment, un changement lexicographique sur les pages internet du dictionnaire allemand Duden (l'un des plus connus dans l'espace germanophone) a fait beaucoup de vagues. Auparavant, les formes masculines (p. ex. Student (« étudiant ») étaient définies de manière peu spécifique (" personne qui étudie dans une haute école») et la forme féminine Studentin ("étudiante») renvoyait à cette entrée principale au masculin. Désormais, on trouve régulièrement deux entrées :

-Student: männliche Person, die an einer Hochschule studiert; Studierender ${ }^{8}$ («personne masculine étudiant dans une haute école »)

- Studentin: weibliche Person, die an einer Hochschule studiert; Studierende («personne féminine étudiant dans une haute école »)

Sans le dire ouvertement, ce dictionnaire a donc opéré un double changement: d'une part, il ne considère plus, dans le domaine des noms communs de personne, les formes féminines comme (formellement et/ou sémantiquement) secondaires par rapport aux formes masculines correspondantes, mais il présente les deux dans des entrées séparées ${ }^{9}$. D'autre part, il supprime aussi, sans l'avoir marqué explicitement, la fonction générique des noms masculins, au moins dans leur description sémantique (cf. « personne étudiant » - > « personne masculine étudiant »).

Bien d'autres observations et régularités ont été faites à propos de la généricité du masculin. Ainsi, Cerquiglini (2018) distingue entre divers usages au singulier et au pluriel, en postulant p. ex. (p. 64) : «Le masculin générique pluriel s'emploie lorsqu'il n'est pas nécessaire, ou utile, ou désirable, de signaler que le groupe humain est 
sexuellement mixte : quand une explicitation des genres n'est pas de mise. » Mais est-il toujours possible de décider du nécessaire, de l'utile et du désirable - et peut-on présumer que d'autres font les mêmes choix?

8 L'histoire du masculin à valeur générique est d'ailleurs relativement mal étudiée, notamment en ce qui concerne l'état de la langue avant les premières codifications grammaticales. Si la règle de Vaugelas, selon laquelle « le genre masculin estant le plus noble, doit predominer toutes les fois que le masculin \& le feminin se trouuent ensemble ( Vaugelas 1647: 83), a souvent été mentionnée et discutée de manière critique (cf. p. ex. Viennot 2014), il n'est pas simple d'évaluer correctement les usages du passé, et cela jusqu'aux temps modernes: qu'a-t-on utilisé pour désigner des groupes de personnes inconnues, mixtes, etc. ? Souvent, ces groupes désignés au moyen de formes masculines se composaient de facto majoritairement (voire uniquement) d'hommes, et même là où l'on peut présumer l'existence de groupes mixtes (les enfants, les personnes habitant un lieu ou un pays, etc.), il est probable que la perspective adoptée ait souvent été majoritairement androcentrée. Il est ainsi difficile de savoir quelle a été historiquement l'influence mutuelle entre inégalités sociales et inégalités langagières. Et n'oublions pas que nombre de formes féminines ont certes existé dans l'ancienne langue (p. ex. le Livre des métiers de la fin du fin XII ${ }^{\mathrm{e}}$ siècle, Boileau 1980), mais que nombreuses sont aussi celles qui ont dû être recréées ou redécouvertes au XXe siècle.

9 Aujourd'hui, la mixité est réelle dans beaucoup de contextes et il existe des formes masculines et féminines pour la plupart des noms communs de personne - même s'il peut y avoir des variantes (sculpteuse ou sculptrice?, prédécesseuse, prédécesseure ou prédécessrice ?). La question de la généricité se pose donc d'autant plus clairement pour les noms masculins. Si elle n'est pas inscrite grammaticalement dans la sémantique de chaque forme masculine, elle est alors sujette à des négociations et inférences.

\section{Toutes pour une}

10 Les guides de langue non sexiste / inclusive recommandent en général de renoncer aux formes génériques, au profit d'autres stratégies, qu'on peut résumer comme suit :

- les stratégies de visibilisation préconisent en général les doubles formes féminine et masculine (en entier ou de forme abrégée) ;

- par la neutralisation, on entend l'évitement de formes génériques, par l'usage de noms épicènes ou de désignations qui ne différencient pas entre formes féminine et masculine ;

- enfin, parfois, on procède par évitement de noms communs de personnes, en les substituant par d'autres formulations.

11 Dans le tableau qui suit, je donne une vue d'ensemble sur quelques stratégies d'évitement de formes masculines à valeur générique ${ }^{10}$ :

\begin{tabular}{|l|l|l|}
\hline & dédoublement & $\begin{array}{l}\text { les lectrices et lecteurs } \\
\text { ils et elles }\end{array}$ \\
\cline { 2 - 3 } $\begin{array}{l}\text { visibilisation (de la forme } \\
\text { féminine) }\end{array}$ & $\begin{array}{l}\text { les lecteurs/trices } \\
\text { les lecteurs.trice.x.s } \\
\text { ils.elles }\end{array}$ \\
\hline
\end{tabular}




\begin{tabular}{|l|l|l|}
\cline { 2 - 3 } & féminin à valeur générique & $\begin{array}{l}\text { les lectrices } \\
\text { elles }\end{array}$ \\
\hline \multirow{4}{*}{ neutralisation } & formes épicènes & $\begin{array}{l}\text { les touristes } \\
\varnothing\end{array}$ \\
\cline { 2 - 3 } & formes non différenciées & les personnes (qui...) \\
\cline { 2 - 3 } & formes neutralisantes nouvellement & $\begin{array}{l}\text { les lectaires } \\
\text { iel(s), illes, ellui, toustes, } \\
\text { celleux }\end{array}$ \\
\hline \multirow{2}{*}{ évitement } & noms collectifs & le lectorat \\
\cline { 2 - 4 } & reformulations & $\begin{array}{l}\text { Si vous lisez, ... } \\
\text { Lors de la lecture,... }\end{array}$ \\
\hline
\end{tabular}

Quelques observations peuvent se faire par rapport à ce tableau :

- toutes ces stratégies peuvent être considérées comme relevant de l'écriture non sexiste/ inclusive, mais seulement une partie d'entre elles (notamment celles qui sont marquées en jaune ou en rose) sont particulièrement saillantes - et controversées dans le débat sur l'écriture inclusive ;

- certaines solutions sont plus explicites quant à leur finalité que d'autres, dont la valeur « inclusive » peut rester plus ou moins implicite ;

- on peut se demander si toutes les stratégies sont compatibles entre elles : les avis divergent concernant certains principes de base, p. ex. : peut-on mélanger ou non des doublets avec des formes à visée générique? Peut-on alterner entre des masculins à valeur générique et des féminins à valeur générique ? $^{11}$

\section{Une pour toustes?}

13 Il semble clair qu'actuellement, aucune stratégie n'emporte l'adhésion de tout le monde: certaines sont rejetées pour leur nouveauté; d'autres pour des raisons de stylistique (p. ex. les suites de doublets) ou de lisibilité des textes (certaines formes abrégées). Si toutes poursuivent un but commun (l'évitement partiel, voire complet du masculin à valeur générique), elles s'y prennent de manières bien diverses, et parfois avec des signes (abréviatifs) différents les unes par rapport aux autres. Pour celles et ceux qui souhaitent disposer de règles simples et universellement applicables (et pourquoi pas: enseignables), ce constat n'est guère satisfaisant. Il est ainsi probable qu'on voie à l'avenir plusieurs moyens de gérer les noms communs de personne.

Beaucoup ne verront pas l'utilité de changer leurs habitudes et maintiendront des formes masculines à valeur générique, qui ont encore la caution de nombreuses grammaires (et génèrent des inférences connues, quoique contestées).

D'autres seront convaincu'e's de la nécessité de pratiques alternatives et adopteront la stratégie préférentielle qui convient le mieux pour leurs usages, prêt·e·s à défendre leurs choix. 
16 La position la plus difficile sera celle des personnes qui n'ont pas d'avis très prononcé pour ou contre l'écriture inclusive ou qui n'ont pas le libre choix des stratégies: typiquement des personnes employées dans une administration ou qui sont amenées à travailler avec des textes rédigés par d'autres ${ }^{12}$ : pour elles, l'absence de règles universellement acceptées se fait le plus difficilement sentir. Certaines conventions qui ont été proposées ces dernières années (notamment sous le libellée d'«écriture inclusive »), impliquant des formes peu habituelles (comme agriculteurs.rices ou ils·elles) et ne présentant pas encore de solution claire pour les enjeux de la non-binarité, n'auront peut-être qu'une durée de vie moyenne.

17 Ainsi, une seule stratégie ne convient pour l'instant pas à tous / toutes / toutes et tous / toustes (veuillez choisir ce qui vous convient le mieux). Peut-on s'en exciter ? Oui. Mais doit-on s'en inquiéter? À mon avis : non, dans le grand jardin de la langue il y a de la place pour faire coexister divers usages.

\section{BIBLIOGRAPHIE}

BOILEAU, Etienne. 1980. Le Livre des métiers: XIIIe siècle. LESPINASSE, René de \& BONNARDOT, François (éd.). Genève : Slatkine Reprints (réimpression de l'édition de Paris, 1879).

CERQUIGLINI, Bernard. 2018. Le ministre est enceinte ou la grande querelle de la féminisation des noms. Paris : Éditions du Seuil

ELMIGER, Daniel. 2015. «La répétition de noms communs de personnes pour éviter le masculin à valeur générique ». Le discours et la langue 7(2) MAGRI-MOURGUES, Véronique \& RABATEL, Alain (éd.), numéro spécial « La répétition et les genres » : 97-112.

GYGAX, Pascal, ZUFFEREY, Sandrine \& GABRIEL, Ute. 2021. Le cerveau pense-t-il au masculin? Cerveau, langage et représentations sexistes. Paris : Le Robert.

LOBIN, Henning. 2021. « Wo genau ist das Generische im generischen Maskulinum? ». SciLogs. Berlin : Dudenverlag. https://scilogs.spektrum.de/engelbart-galaxis/wo-genau-ist-dasgenerische-im-generischen-maskulinum/

MICHEL, Lucy. 2016. La relation entre genre grammatical et dénomination de la personne en langue française. Approches sémantiques. Thèse de doctorat en sciences du langage. Dijon : Université de Bourgogne.

VAUGELAS, Claude Favre de. 1647. Remarques sur la langue françoise : utiles à ceux qui veulent bien parler et bien escrire. Paris : Jean Camusat \& Pierre Le Petit

VIENNOT, Eliane. 2014. Non, le masculin ne l'emporte pas sur le féminin ! Petite histoire des résistances de la langue française. Donnemarie-Dontilly : Éditions iXe 


\section{NOTES}

1. Devoir expliquer un titre, c'est un peu comme devoir expliquer une blague : on gagne peut-être en clarté, mais on risque de perdre la chute. Mais allons-y :

toutes : toutes les stratégies (pour éviter les formes masculines à valeur générique)

pour une : pour une visée commune

une : une seule stratégie

pour toustes : pour toute personne, toutes et tous.

Cela dit : je crains que pour comprendre le titre, il ne faille lire la chronique en entier...

2. Cf. la chronique 8 ( $Y$ Y a-t-il un guide dans la rédaction?»).

3. Aujourd'hui, on doit chercher davantage pour trouver des formes controversées : peut-être autrice ou successeuse/successrice? (Et lançons un défi : pourquoi pas la marine et la médecine pour désigner des femmes?...).

4. Ce n'est pas tout, bien sûr : il y va aussi d'autres sortes d'inégalités, stéréotypes, etc. en lien avec la représentation langagière des humains, dont je fais abstraction ici.

5. Cf. Gygax et al. (2021) pour la recherche psycholinguistique à ce sujet.

6. Rappelons qu'ici, il n'est pas question de référence (généralisante vs individualisante), où l'on parle également de généricité (vs spécificité), en linguistique (cf. la chronique «Le féminin générique ou : une généricité peut en cacher une autre »).

7. Ces calculs inférentiels sont explicités en détail dans Charaudeau (2021), qui semble présumer qu'il y a une (seule) façon juste de les faire.

8. Les deux formes participiales Studierender et Studierende (littéralement "étudiant", " étudiante ») sont elles aussi controversées. Tantôt différenciées formellement (dans certains usages au singulier), tantôt indifférenciées (les formes plurielles neutralisent toujours la différenciation féminin/masculin), elles sont souvent récusées parce qu'au contraire de Student/ Studentin, elles ne désigneraient que des personnes étant en train d'étudier - et non pas l'étudiant·e qui maintient sa "fonction" même en dehors des salles de cours et du travail individuel. Ce point de vue est hautement discutable - et même difficile à saisir si l'on l'observe du point de vue francophone, où de pareils doublets n'existent guère.

9. Cf. à ce sujet la thèse de Lucy Michel (2016).

10. Pour une autre présentation des stratégies, cf. Elmiger (2015).

11. Ce qui semble inconcevable pour beaucoup relève d'une évidence pour d'autres; ainsi, dans certains textes anglophones, on alterne régulièrement entre masculins et féminins à valeur générique, p. ex. d'une page ou d'un chapitre à l'autre.

12. Cf. la chronique « Pour ou contre l'écriture inclusive : l'injuste milieu?».

\section{RÉSUMÉS}

En général, la critique sur laquelle se basent les guides de langue non sexiste / inclusive concerne les noms masculins à valeur générique. Cette fonction générique est-elle inscrite dans la grammaire, négociée discursivement ou le fruit d'un calcul inférentiel? Toute une série de (méta)stratégies ont été proposées pour éviter les formes masculines à valeur générique. Si ainsi, toutes ces stratégies poursuivent un même objectif, on peut aussi observer qu'il n'y en a pas une seule qui trouve l'adhésion de tout le monde. 
In general, the criticism on which non-sexist / inclusive language guides are based concerns masculine nouns with generic value. Is this generic function embedded in the grammar, discursively negotiated or the result of an inferential calculation? A whole series of (meta)strategies have been proposed to avoid generically intended masculine forms. If all these strategies have the same objective, it can also be observed that there is no single one that is universally accepted.

\section{INDEX}

Thèmes : Chroniques

Mots-clés : genre grammatical, masculin générique, stratégies, diversité

Keywords : grammatical gender, generic masculine, strategies, diversity 